\title{
Effects of Cavity Diameter on Acoustic Impedance in a Complex Acoustic Environment
}

\author{
M. C. Brown* and M. G. Jones ${ }^{\dagger}$ \\ NASA Langley Research Center, Hampton, VA 23681 \\ 24. $4^{\text {th }}$ AIAA/CEAS Aeroacoustics Conference \\ 25-29 June 2018; Atlanta, GA
}

\begin{abstract}
This paper investigates the effects of cavity diameter on the acoustic performance of conventional perforate-over-honeycomb liners. This investigation is a follow up to a previous study where it was demonstrated that the cavity diameter has a relatively small effect on the acoustic impedance for core cavity diameters up to 2.0 inches. In the current study, liner concepts are exposed to a complex acoustic environment, where higher-order modes are present. Four liner configurations are explored in this investigation, which include two cavity cores of different cavity diameters combined with two facesheets. These configurations are tested in the Grazing Flow Impedance Tube (GFIT) at Mach 0.0, 0.3, and 0.5 at a source sound pressure level of $150 \mathrm{~dB}$. A comparison of the acoustic performance of these samples suggests that the cavity diameter has a relatively small effect on the acoustic impedance for core cavity diameters up to 2.0 inches at these conditions. These configurations are exposed to a more complex acoustic environment (e.g., higher-order modes) in the Curved Duct Test Rig (CDTR) at Mach 0.0 and 0.3.
\end{abstract}

\section{Introduction}

For over four decades, acoustic liners have be an effective means of suppressing fan tone noise propagating through inlet and exhaust ducts of commercial aircraft turbofan engines. During this time, the bypass ratio of these engines has increased, which has caused a decrease in the ratio of length of the liner to the diameter of the duct. This has caused the effectiveness of acoustic liners to be reduced and has, therefore, resulted in a renewed interest in the exploration of novel liner configurations that might offer the potential for improved acoustic absorption. Also, it has become increasingly important to include liners wherever possible throughout the engine nacelle.

Current aircraft engine nacelles typically have acoustic liners mounted in the inlet and the outer walls of the aft bypass duct. They are also sometimes implemented in the inner walls of the aft bypass duct. However, this latter usage is often avoided due to manufacturing issues. The proximity to the core exposes inner wall liners to elevated temperatures, thus affecting the choice of bonding material (glue) used in the liner construction. Also, the weight of the inner wall liners (and the associated performance implications) often outweighs the benefits of the additional acoustic absorption they provide.

The current paper is a follow up to a previous paper ${ }^{1}$ that explored the effects of modifying the geometry of these inner wall liners on noise attenuation in a simple acoustic environment. In the previous paper, configurations were explored in which the core cavity diameter was increased, such that the liner contained fewer cavities and, thus, fewer partitions between cavities. This design offered the potential to decrease the weight due to the reduced number of partitions. The study was conducted in the NASA Langley Grazing

\footnotetext{
*Research Engineer, Research Directorate, Aeroacoustics Branch, Mail Stop 164D; Martha.C.Brown@nasa.gov.

${ }^{\dagger}$ Senior Research Scientist, Research Directorate, Structural Acoustics Branch, Mail Stop 463; Michael.G.Jones@nasa.gov.
} 
Flow Impedance Tube, with source sound pressure levels of 120 and $140 \mathrm{~dB}$ and at two flow conditions, Mach 0.0 and 0.3. It should be noted that results were achieved in a simple acoustic environment where only plane waves exist in the hard wall sections upstream and downstream of the liner. Preliminary results showed that cavity diameter had a minimal effect on the acoustic impedance spectra. The results of this research were well received in the aeroacoustics community; however, there was a desire to extend the test matrix to $150 \mathrm{~dB}$ sound pressure level and mean flow velocity of Mach 0.5. The results of this extended test matrix are presented in this paper, where the samples were tested at $150 \mathrm{~dB}$ and at mean flow velocities of Mach 0.0, 0.3 and 0.5.

The effects of exposure to a more complex acoustic environment (i.e., higher order modes) are also explored via tests in the NASA Langley Curved Duct Test Rig (CDTR). The CDTR utilizes two arrays of microphones mounted upstream and downstream of the liner test section to determine the modal structure of the sound in the duct. Acoustic performance will be assessed by determining whether or not the target mode attenuation for the largest (LC3) core cavity diameter liner is similar to the target mode attenuation for the smallest (LC1) core cavity diameter for the same facesheet.

The remainder of this paper is organized as follows: Section II provides a description of the Experimental Methods employed in this study. Methods of assessing Acoustic Performance are provided in Section III, Results and Discussion are provided in Section IV, and Concluding Remarks are provided in Section V.

\section{Experimental Methods}

\section{A. GFIT Evaluation Liners}

The liners used in this study are composite structures consisting of a perforated facesheet, a square-shaped core, and a rigid back plate. Two (2) cores with square-shaped cavities and a fixed cavity depth of 1.5 in and varying core cavity diameters were fabricated with two (2) facesheets of varying porosities. Each GFIT core and facesheet was constructed via sterolithography at NASA Langley, which allowed multiple sample configurations to be constructed in a few weeks at a fraction of the cost in comparison with conventional fabrication methods. The two core cavity diameters are: 0.40 in $\mathrm{x} 0.40$ in (designated by LC1) and 2.00 in $\mathrm{x}$ 2.00 in (designated by LC3). The two facesheet porosities are $4 \%$ and $16 \%$ open area (OA). Each facesheet has a hole diameter of 0.040 in and a thickness of 0.030 in. For each facesheet, the physical location of each perforate hole is arranged so that it is not obstructed due to a core partition wall. The nomenclature of the test matrix is summarized in Table 1. Each GFIT liner sample is 18 in long, with the active surface length being 16.923 in. Each liner configuration was tested at $150 \mathrm{~dB}$ for flow speeds of Mach 0.0, 0.3, and 0.5.

Figure 1 is a photograph of the two GFIT liner cores of different core cavity diameters: LC1 and LC3. Results of the smallest (FS1, 4\% OA) and largest porosities (FS4, 16\% OA) for the GFIT samples will be presented in this paper.

\section{B. Grazing Flow Impedance Tube (GFIT) Test Rig}

The Grazing Flow Impedance Tube (GFIT, see Fig. 2) is used to evaluate the acoustic performance of each liner. The GFIT has a cross-sectional geometry of 2.0 in wide by 2.5 in high, such that higher-order modes in the horizontal and vertical dimensions cut-on at different frequencies. It allows evaluation of acoustic liners with lengths from 2 in to $24 \mathrm{in}$. The surface of the test liner forms a portion (16.923 in active length for this investigation) of the upper wall of the flow duct. For this investigation, the source section consists of twelve acoustic drivers mounted upstream (exhaust mode) of the test section. These drivers are used to generate tones (one frequency at a time) at a source sound pressure level (SPL) of $150 \mathrm{~dB}$ over a frequency range of $600 \mathrm{~Hz}$ to $3000 \mathrm{~Hz}$, in increments of $200 \mathrm{~Hz}$. Flow speeds of Mach 0.0, 0.3 and 0.5 will be presented in this paper.

Fifty-seven flush-mounted microphones located in the lower wall (opposite the liner) are used to measure the acoustic pressure field over the axial length of $0 \leq x \leq L$ (see Fig. 3). Note the leading edge of the liner is 8.25 in $(x=8.25$ in $)$ from the source plane. For each data acquisition, 1000 averages on each microphone channel (1024 data points per average, at a sample rate of 25,600 Hz) are taken. A cross-spectrum signal extraction method ${ }^{2}$ is used to determine the amplitudes and phases at each of the microphone locations relative to the amplitude and phase at the reference microphone location. Results from the microphones on 
the lower wall are used to educe the uniform acoustic impedance of that liner operating at a specific flow condition.

The impedance eduction method employed in this paper is based on the Prony method. ${ }^{3}$ The Prony method solves a linear system of equations formed from the complex acoustic pressures measured with the microphones located on the wall opposite the liner. The coefficients derived are used to create a polynomial equation, and the complex roots of that polynomial are used to educe the impedance of a liner. The liner is assumed to be locally-reacting, and the mean flow is assumed to be uniform for this method. Acoustic resistance, $\theta$, and reactance, $\chi$, are normalized with respect to $\rho c$, where $\rho$ is the density and $c$ is the speed of sound.

\section{CDTR Evaluation Liners}

The CDTR evaluation liners used in this study have the same characteristics as the GFIT evaluation liners except for overall physical size; the active liner size is 30.08 in long x 13.99 in wide. The core is constructed via $3 \mathrm{D}$ printing at NASA Langley using polycarbonate material, which ensures strength and durability in a realistic wind tunnel environment. The facesheets are constructed by machining the perforate hole pattern on a G10 fiberglass sheet. G10 material was selected due to its strength and ability to bond to the polycarbonate core via a water soluble adhesive. An aluminum frame provides additional strength while allowing the panel to be installed into the test section easily in a few minutes. Core/facesheet combinations are easily interchanged by immersing the liner sample (facesheet down) in hot tap water which allows the water soluable adhesive to dissolve easily. The facesheet easily separates from the core, where any residual adhesive is removed before securing the facesheet to another core panel. Figure 4 is a photograph of one of the CDTR panel configurations, LC1FS4 ( 0.4 in x 0.4 in core, $16 \%$ OA facesheet). Figure 5 is a close-up view of the same CDTR panel that allows the facesheet to be seen more clearly. Results for the $4 \%$ OA and $16 \%$ OA facesheet configurations are presented in this paper.

\section{Curved Duct Test Rig (CDTR)}

The Curved Duct Test Rig (CDTR) is an experimental facility designed to assess the acoustic and aerodynamic performance of aircraft engine nacelle liners (Fig. 6). The CDTR has a cross sectional geometry of 6 in wide by 15 in high. The test section size is between $25 \%$ and $100 \%$ of the scale of the aft bypass ducts of aircraft engines ranging in size from a business jet to a large passenger jet. The CDTR is an open loop wind tunnel that uses a fan to draw unconditioned atmospheric air through the test section. Details have been described in previous papers. ${ }^{4,5}$

Sound is generated in the test section of the CDTR by an array of 32 loudspeakers. The maximum tonal sound level that can be generated in the duct is on the order of $140 \mathrm{~dB}$, with the amplitude of the desired mode typically at least $10 \mathrm{~dB}$ above the amplitude of all other modes for all frequencies of interest. Details of the noise generation mechanism and control system are described in a previous paper. ${ }^{6}$ For this test, tones are typically generated at $138 \mathrm{~dB}$ from 600 to $3000 \mathrm{~Hz}$ at $200 \mathrm{~Hz}$ increments, where the mode is expressed as (Horizontal, Vertical).

The CDTR utilizes a total of 158 microphones, where 63 are mounted upstream of the liner, 63 are mounted downstream of the liner, and 32 are mounted in the lid of the liner test section. The results presented here are based solely on the upstream and downstream microphone arrays. For each data acquisition, 1000 averages on each microphone channel (1024 data points per average, at a sample rate of 25,600 $\mathrm{Hz}$ ) are taken. A cross-spectrum signal extraction method ${ }^{2}$ is used to determine the amplitudes and phases at each of the microphone locations relative to the amplitude and phase at the reference signal. Measurements from the upstream and downstream arrays are used to determine modal structure of the sound in the duct, such that the effects of cavity diameter on transmisson loss can be investigated. Four target modes $(\mathrm{H}, \mathrm{V})$ will be examined in this investigation: $(0,0),(0,1),(1,0)$, and $(1,1)$. Tests will be conducted under two flow regimes: Mach 0.0 and 0.3 . 


\section{Acoustic Performance}

For the results in the GFIT, a comparison of acoustic impedance spectra educed for the small and large cavity diameter samples is used to assess cavity diameter effects. The acoustic impedance is an intrinsic parameter that is commonly used to determine how well a liner will absorb sound when placed in different aeroacoustic environments (e.g., in the walls of an aircraft engine nacelle). This comparison is evaluated with data acquired without (Mach 0.0) and with (Mach 0.3 and 0.5) flow at a source pressure level of $150 \mathrm{~dB}$.

For the results in the CDTR, a comparison of transmission loss spectra measured with the small and large cavity diameter samples, is used to assess the effects of cavity diameter. This comparison is evaluated without (Mach 0.0) and with (Mach 0.3) flow at a source pressure level of $138 \mathrm{~dB}$. Results are limited to those for which the target mode is dominant, i.e., for those frequencies where the target mode upstream of the liner is at least $10 \mathrm{~dB}$ above all other modes.

\section{Results and Discussion}

Figures 7 and 8 compare the acoustic impedance, $Z$ (where, $Z=\theta+i \chi$ ), spectral results from the GFIT for small (LC1) and large (LC3) cavity diameter cores, at facesheet porosities $4 \%$ OA (FS1) and 16\% OA (FS4), respectively. Flow regimes are Mach 0.0, 0.3, and 0.5.

Figure 7 presents results for LC1 and LC3 cores with a 4\% OA facesheet, at $150 \mathrm{~dB}$. Note that increasing the cavity diameter core has minimal effect on acoustic resistance for the three flow regimes. However, there is a noticable effect on acoustic reactance, especially at the lower frequencies. The reactance values are higher (closer to zero) for the large cavity than for the small cavity for frequencies below resonance, but are nearly identical for both cavity diameters for frequencies above resonance. This "flattening" of the reactance causes the large cavity liner to provide more broadband absorption, which is of potential advantage for the liner designer.

Figure 8 provides the corresponding results for the LC1 and LC3 cores with a 16\% OA facesheet, at $150 \mathrm{~dB}$. Again, increasing the cavity diameter core has minimal effect on acoustic resistance for all three flow regimes. As noted in Fig. 7, there is a slight effect on acoustic reactance, where the spectra is more "flat." In general, the trends for the $16 \%$ OA (FS4) facesheet are quite similar to those observed with the $4 \%$ OA (FS1) facesheet.

Overall, the difference for liners with small and large diameter cavities are sufficiently small to suggest that conventional models previously developed for small diameter cavities could be easily modified to account for these large cavity diameter effects. However, it is important to note that this preliminary conclusion is based on GFIT data for which the acoustic environment is quite simple (limited to plane waves in the hard wall sections of the duct). The following results explore the effects of exposing these samples to a more complex acoustic environment in the CDTR.

Figures 9, 10 and 11 are the acoustic results from the CDTR at Mach 0.0 and 0.3. Figure 9 displays the upstream modal content for liner core LC1, 4\% OA at Mach 0.0 for target modes $(0,0),(0,1),(1,0)$ and $(1,1)$. Note that there is at least $10 \mathrm{~dB}$ mode separation (difference between the target mode and the next closest mode) for the $(0,0)$ target mode for frequencies below $2000 \mathrm{~Hz}$. For the $(0,1)$ target mode, clear mode separation is achieved for frequencies below $2400 \mathrm{~Hz}$. There is sufficient target mode separation for modes $(1,0)$ and $(1,1)$ over the entire frequency range. These plots only display modes of interest for clarity. Assuming uniform flow and that the modes are orthogonal, it is presumed that a mode will scatter into another mode with the same vertical index. For example, the plane wave, $(0,0)$, will scatter into $(1,0)$ or $(2,0)$; target mode $(1,0)$ will scatter into $(1,0)$ or $(2,0)$; target mode $(0,1)$ will scatter into $(1,1)$ or $(2,1)$; target mode $(1,1)$ can scatter into $(2,1)$ or $(0,1)$ modes.

Figure 10 is a comparison of liner cores LC1 and LC3 for a 4\% OA facesheet, operating at target mode $(0,0)$, Mach 0.0. Figure 10a shows the sound source spectra for LC1, and Fig. 10b shows the sound source spectra for LC3. Note in both figures, a red dashed line is drawn to clearly define the frequency where the mode separation is less than $10 \mathrm{~dB}$, and the higher frequencies are shaded out in grey to indicate those results are inconclusive. Figure 10c shows the transmission loss for LC1, and Fig. 10d shows the transmission loss for LC3. Like Figs. 10a and 10b, a red dashed line is drawn to indicate the frequency where mode spearation between target mode and the next highest mode is less than $10 \mathrm{~dB}$, and frequencies to the right of this line are ignorned in the analysis. In Fig 10e, the transmission loss of LC3 is subtracted from LC1. There are two 
components that contriube to the transmission loss of a target mode. The first component is absorption, and the second component is scattering into other modes. This investigaton did not focus on the individual components of the transmssion loss, but on the overall transmission loss of a target mode. Positive values indicate that liner core LC1 attenuated the target mode better than liner core LC3. Negative values indicate that liner core LC3 attenuated the target mode better than liner core LC1.

Recall that the educed acoustic impedance is slightly different for these two cavity diameters, so it is expected for these samples to attenuate the specified target mode slightly differently. However, the similarity of the respective impedance spectra suggests that the mode attenuations should be relatively similar.

Figure 11 is a transmission loss comparison of liner cores LC1 and LC3 with facesheets of porosities $4 \% \mathrm{OA}$ (FS1) and 16\% OA (FS4), for Mach 0.0 and 0.3. Each target mode presented on its spectral plot highlights similarities or differences in the response of liner core LC1 and LC3. If the value is close to zero, then the acoustic field responds to liner core LC3 similarly to liner core LC1. When the 4\% OA is used, the difference in transmission loss for the LC1 and LC3 cores is less than $10 \mathrm{~dB}$ regardless of the source mode. For the $16 \%$ OA facesheet, the results exhibit additional scatter, especially for the no-flow condition. However, the majority of the results are containted within $10 \mathrm{~dB}$ for the two cavity diameters.

\section{Concluding Remarks}

This paper explored the effects of liner core cavity diameter on the acoustic impedance and propagation in a complex acoustic environment. In this study, liner cores with small $(0.4 \mathrm{in})$ and large $(2.0 \mathrm{in})$ cavity diameters were tested in the GFIT at $150 \mathrm{~dB}$, for flow regimes at Mach 0.0, 0.3, and 0.5. Results show that increasing the core cavity had a minimal effect on the acoustic resistance, and a slighty larger effect on the acoustic reactance. Corresponding panels were tested in the CDTR, where modes are present. Transmission losses of these two cores were compared for the same liner configurations.

The effects of cavity diameter are more evident in the presence of higher-order modes, but remain sufficiently well contained to suggest that models previously developed for small diameter cavities could be successfully modified to account for these differences. Nevertheless, there are sufficient differences to warrant further detailed analysis.

A follow on computational study is planned to further evaluate these effects. The impedance spectra from the GFIT data will be used as input to a propagation code to study the response of a single mode (i.e., without the contamination of the other modes).

\section{Acknowledgments}

The authors would like to thank Mr. Alonzo M. Reid for oustanding testing support. This work was funded by the Advanced Air Transport Technology Project of the NASA Advanced Air Vehicles Program.

\section{References}

\footnotetext{
${ }^{1}$ Brown, M. C. and Jones, M. G., "Effects of Cavity Diameter on Acoustic Impedance of Perforate-Over-Honeycomb Liners," AIAA Paper 2017-4189, June 2017.

${ }^{2}$ Bendat, J. S. and Piersol, A. G., Random Data: Analysis and Measurement Procedures, Wiley-Interscience, 1971.

${ }^{3}$ Jones, M. G., Watson, W. R., Howerton, B. M., and Busse-Gerstengarbe, S., "Comparative Study of Impedance Eduction Methods, Part 2: NASA Tests and Methodology," AIAA Paper 2013-2125, May 2013.

${ }^{4}$ Gerhold, C. H., Brown, M. C., Jones, M. G., and Howerton, B. M., "Report on Recent Upgrades to the Curved Duct Test Rig at NASA Langley Research Center," AIAA Paper 2011-2896, June 2011.

${ }^{5}$ Gerhold, C. H., Brown, M. C., Jones, M. G., and Howerton, B. M., "Configuration Effects on Liner Performance," AIAA Paper 2012-2245, June 2012.

${ }^{6}$ Gerhold, C. H., Cabell, R., and Brown, M. C., "Development of an Experimental Rig for Investigation of Higher Order Modes in Ducts," AIAA Paper 2006-2637, 2006.
} 
Table 1: Test Sample Configuration Nomenclature.

\begin{tabular}{|c|c|c|}
\hline \multirow{2}{*}{ Facesheet } & \multicolumn{2}{|c|}{ Core } \\
\cline { 2 - 3 } POA & LC1 & LC3 \\
\cline { 2 - 3 } & $(0.40$ in $\times 0.40$ in $)$ & $(2.00$ in $\times 2.00$ in $)$ \\
\hline FS1 $(4 \%)$ & LC1FS1 & LC3FS1 \\
\hline FS4 $(16 \%)$ & LC1FS4 & LC3FS4 \\
\hline
\end{tabular}

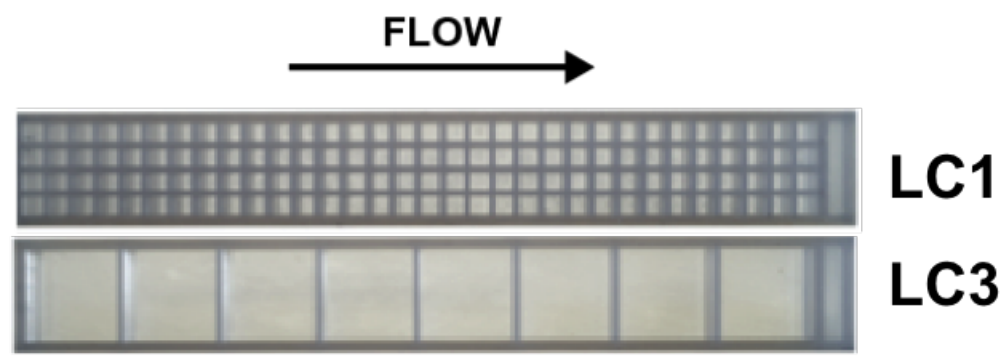

Figure 1: Picture of LC1 and LC3 GFIT cores. Top View.

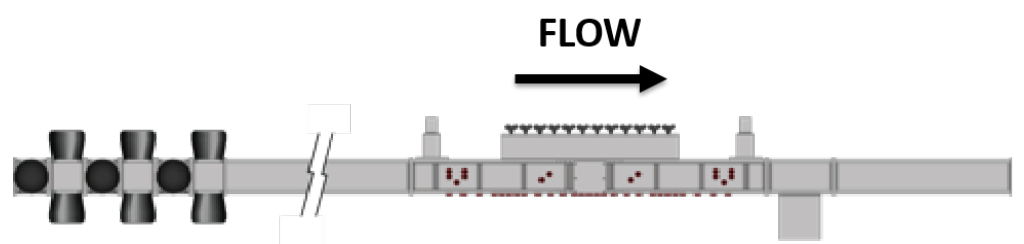

Figure 2: Rendition of Grazing Flow Impedance Tube (GFIT) test section.

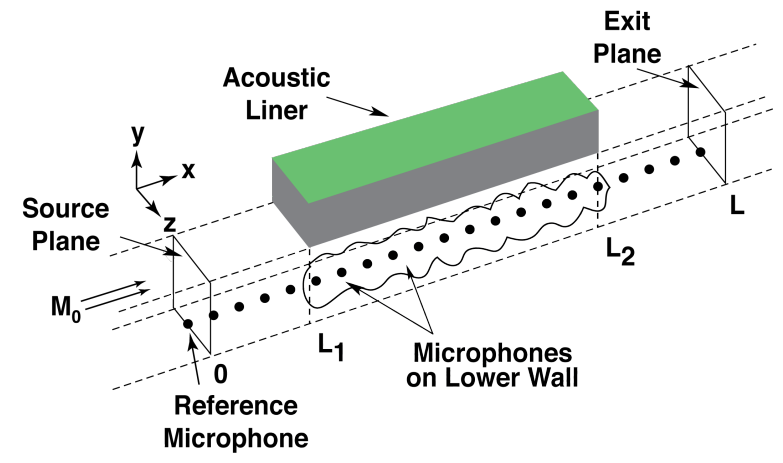

Figure 3: Sketch of Grazing Flow Impedance Tube (GFIT) test section. 


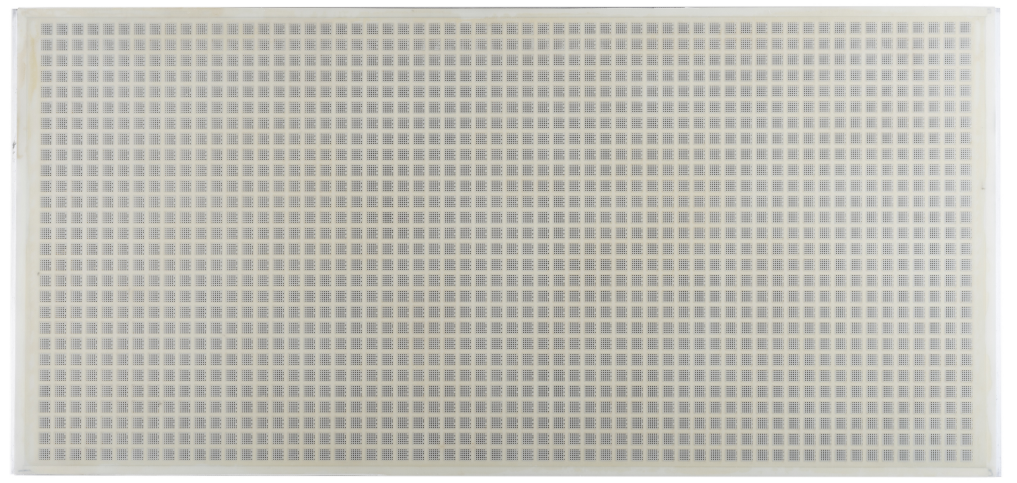

Figure 4: Photograph of CDTR panel, LC1FS4. Top View.

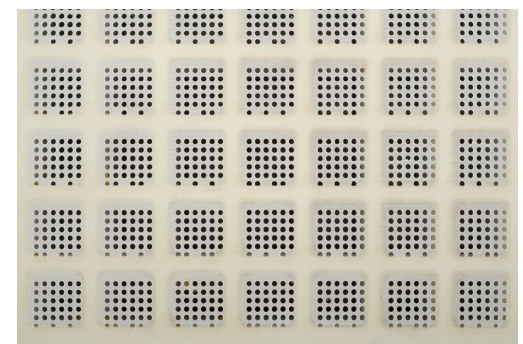

Figure 5: Close-up view of CDTR panel, LC1FS4.

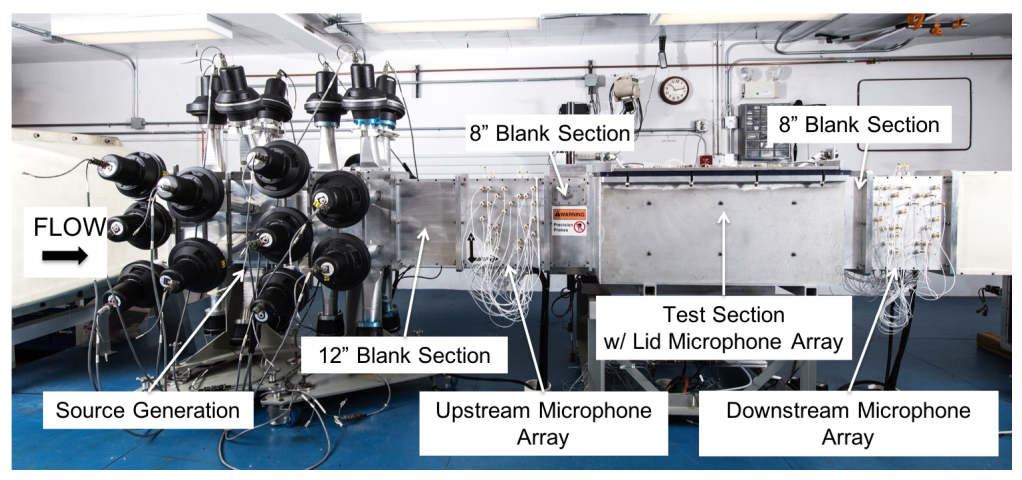

Figure 6: Photograph of Curved Duct Test Rig (CDTR). 


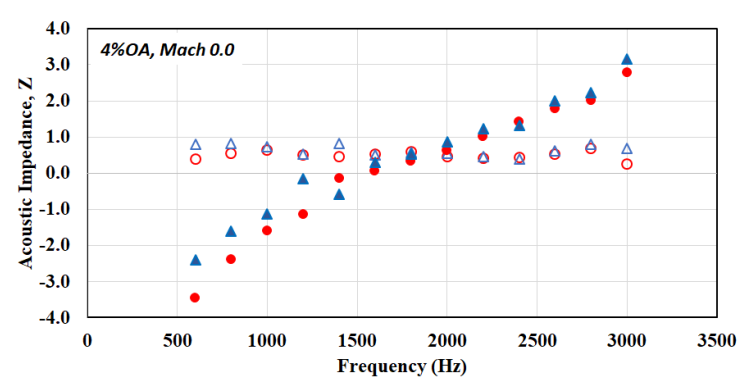

(a) Mach 0.0.

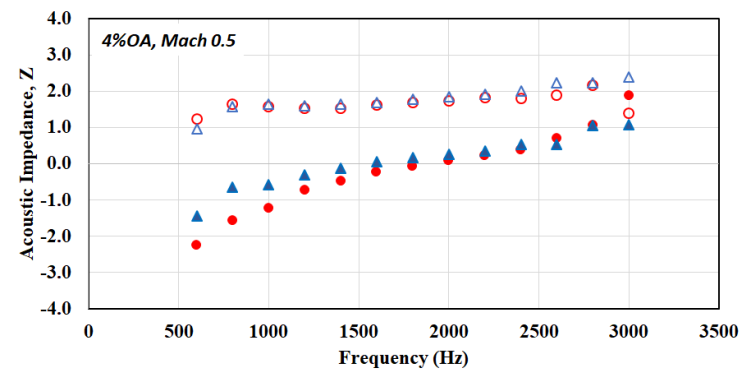

(c) Mach 0.5.

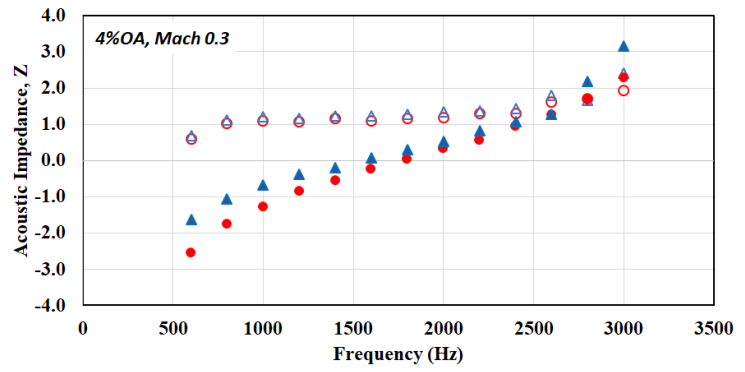

(b) Mach 0.3.

$\circ \theta, \mathbf{L C 1}$

$\triangle \theta, \mathrm{LC3}$

- $\chi, \mathrm{LC1}$

$\triangle \chi, \mathrm{LC} 3$

Figure 7: Acoustic impedance comparison of liner cores LC1 and LC3 with 4\% OA facesheet at $150 \mathrm{~dB}$ in GFIT.

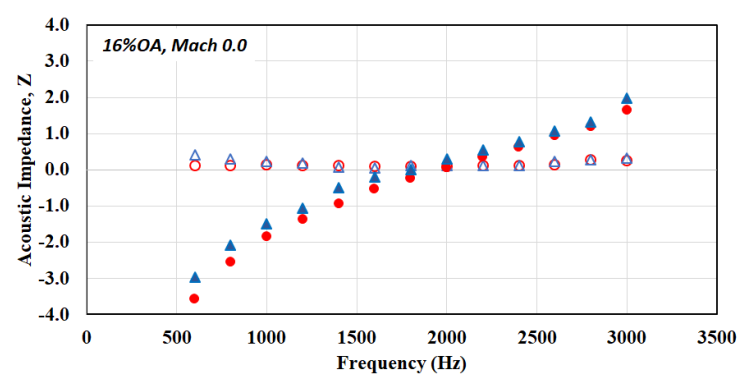

(a) Mach 0.0.

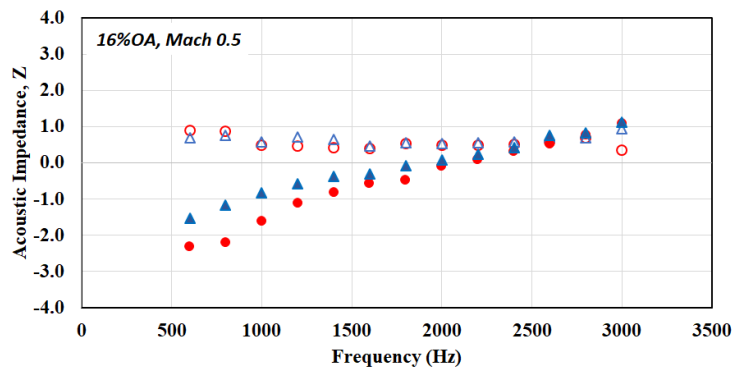

(c) Mach 0.5 .

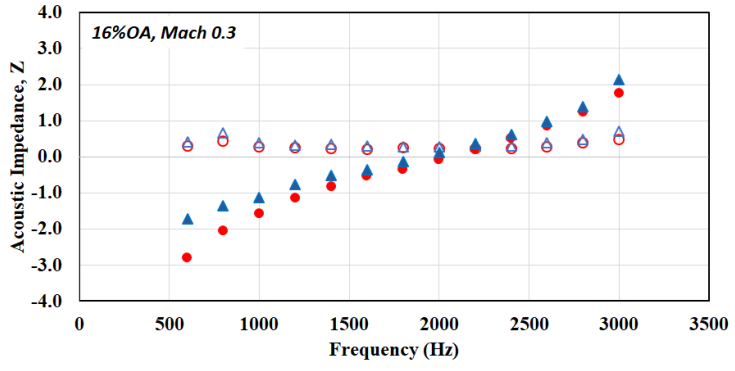

(b) Mach 0.3.

○ $\boldsymbol{\theta}, \mathbf{L C 1}$

$\triangle \theta, \mathrm{LC} 3$

- $\chi, \mathrm{LC} 1$

$\triangle \chi, \mathrm{LC} 3$

Figure 8: Acoustic impedance comparison of liner cores LC1 and LC3 with 16\% OA facesheet at $150 \mathrm{~dB}$ in GFIT. 


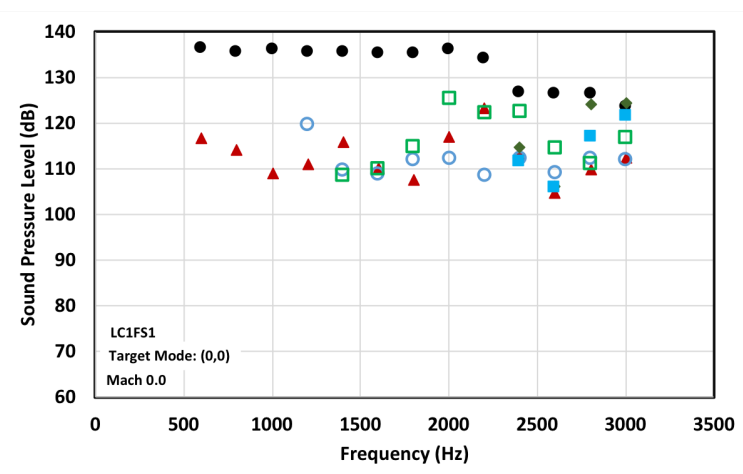

(a) Target Mode $(0,0)$.

Mode $(\mathrm{H}, \mathrm{V}) \bullet 00 \Delta 01$

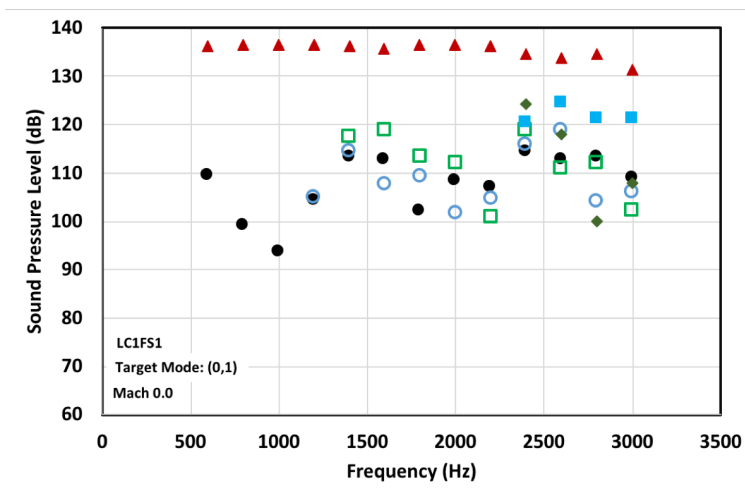

(b) Target Mode $(0,1)$.

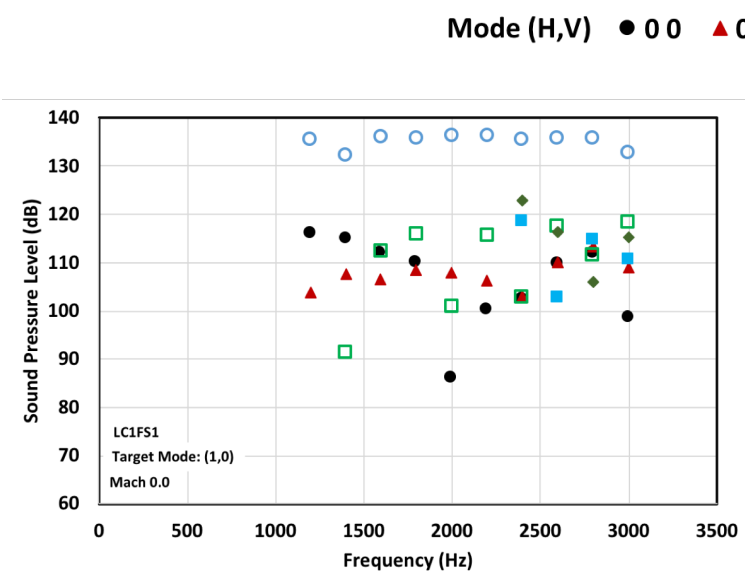

(c) Target Mode $(1,0)$.

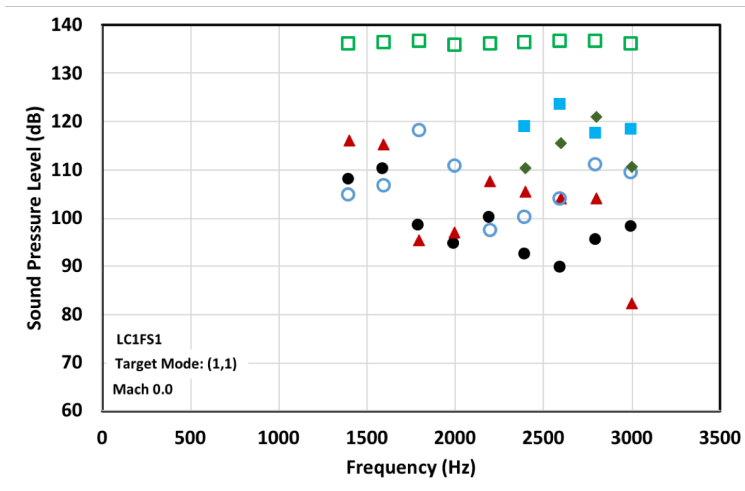

(d) Target Mode $(1,1)$.

Figure 9: Upstream array modal spectra for LC1FS1 with four target modes at Mach 0.0. 


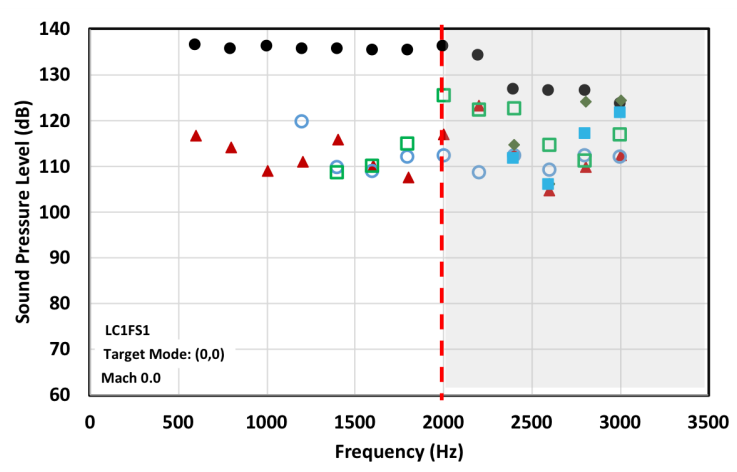

(a) Sound source spectra for LC1FS1.

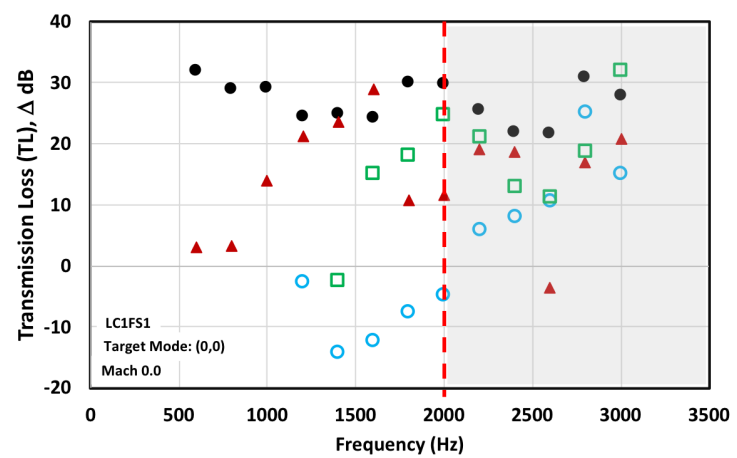

(c) Transmission loss (TL) spectra for LC1FS1.

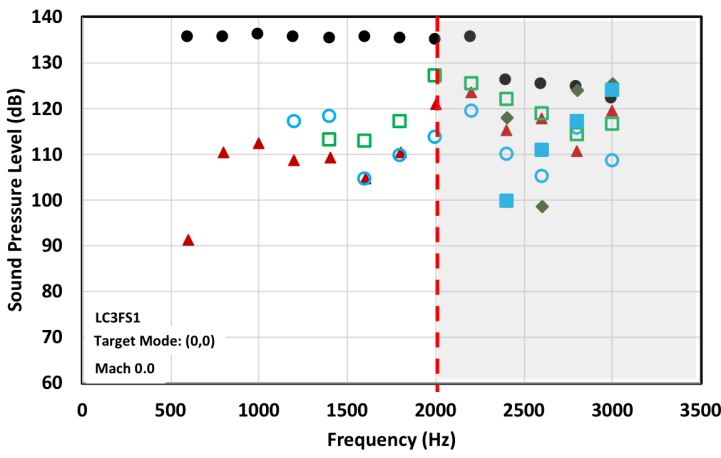

(b) Sound source spectra for LC3FS1.

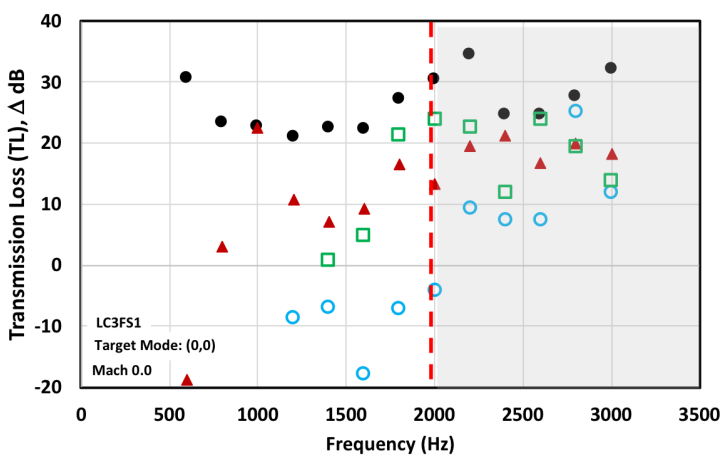

(d) Transmission loss (TL) spectra for LC3FS1.

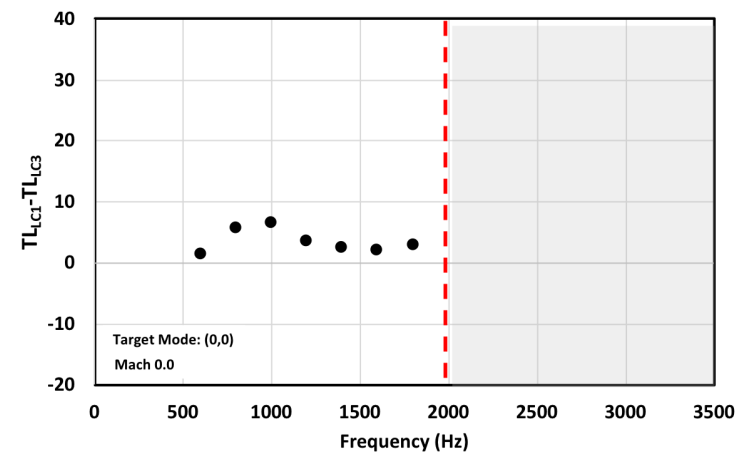
Mode
$(\mathrm{H}, \mathrm{V})$
$\bullet 00$
$\triangle 01$
$\circ 10$
$\square 11$

(e) Transmission loss comparison of LC1 and LC3.

Figure 10: Comparison of liner cores LC1 and LC3 for 4\% OA facesheet, operating at target mode $(0,0)$ and Mach 0.0. 


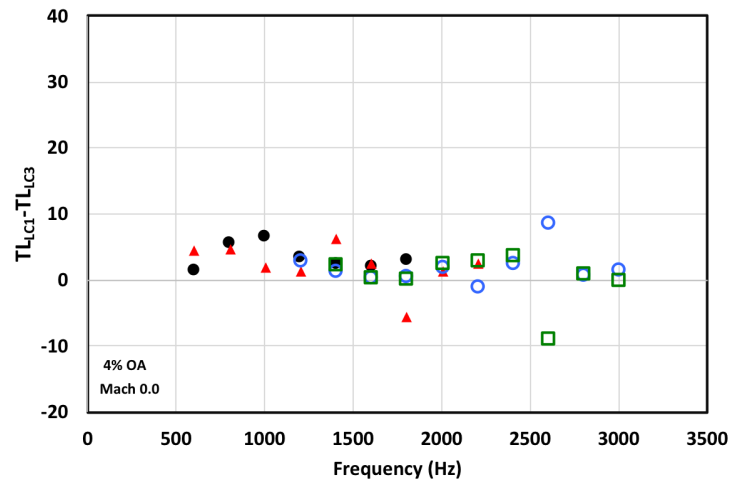

(a) $4 \%$ OA, Mach 0.0.

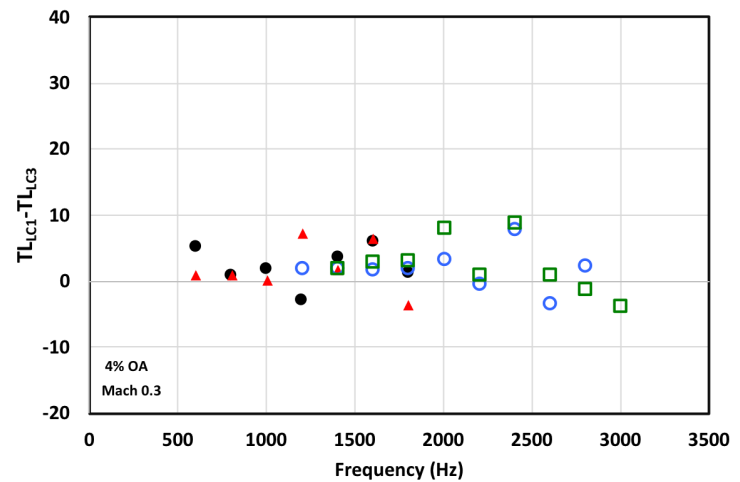

(b) $4 \%$ OA, Mach 0.3 .

\section{Mode $(\mathrm{H}, \mathrm{V}) \bullet 00 \Delta 01 \bigcirc 10 \square 11$}

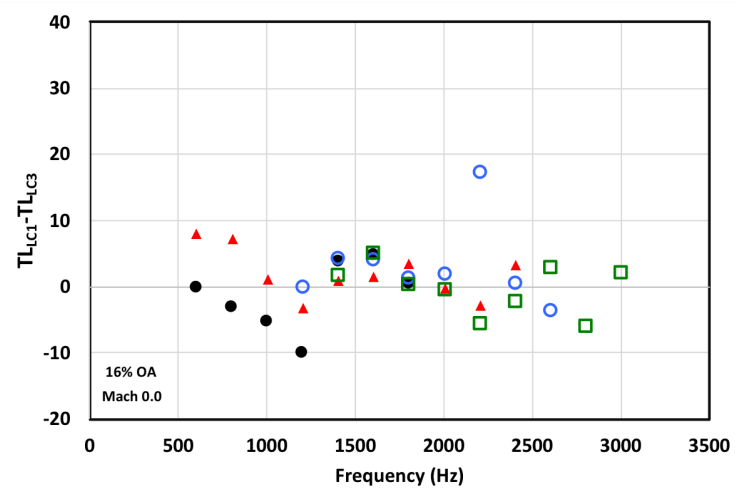

(c) $16 \%$ OA, Mach 0.0 .

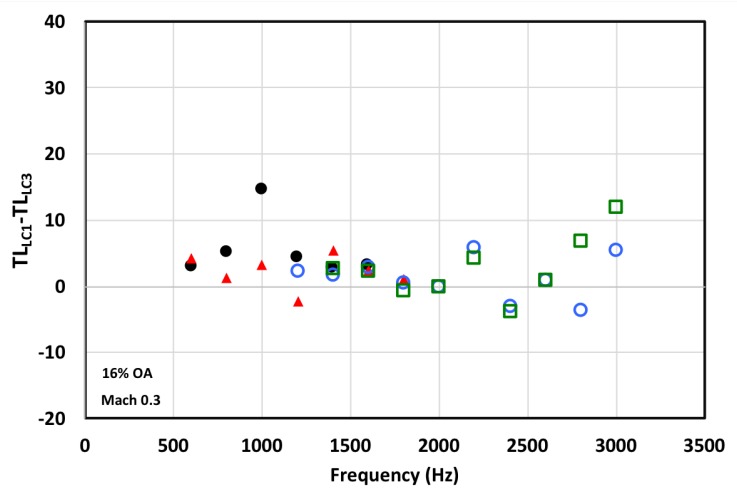

(d) $16 \%$ OA, Mach 0.3 .

Figure 11: Transmission loss comparison of liner cores LC1 and LC3 for $4 \%$ and 16\% OA facesheets and Mach 0.0 and 0.3 . 